\title{
Functional constipation in children - evaluation and management
}

\author{
Magdalena Gibas-Dorna, Jacek Piątek \\ Department of Physiology, Poznan University of Medical Science, Poznan, Poland
}

Prz Gastroenterol 2014; 9 (4): 194-199

DOI: $10.5114 / p g .2014 .45099$

Key words: functional constipation, behavioural treatment, fibre, lactulose.

Address for correspondence: Magdalena Gibas-Dorna MD, Department of Physiology, Poznan University of Medical Science, Poland, 6 Święcickiego St, 60-781 Poznan, Poland, e-mail: physioplus@wp.pl

\begin{abstract}
Almost $1 / 3$ of gastrointestinal consultations are related to constipation, and over $90 \%$ of them are of functional origin. Proper diagnosis and adequate treatment (both nonpharmacological and pharmacological) may limit the number of specialist visits and can be beneficial for therapeutic effects. The treatment is long lasting and requires unique discipline of parents/guardians and coordination supervised by the referring doctor. This paper presents the most important aspects of diagnostic and therapeutic processes regarding functional constipation in neonates and toddlers.
\end{abstract}

\section{Introduction}

Chronic constipation and soiling (encopresis) is a very common health problem among children. According to the available data, the prevalence of constipation in children varies between $1 \%$ and $30 \%$ and accounts for almost 30 percent of consulting visits to a gastroenterologist [1, 2]. Most cases (90-95\%) are functional in origin, and they are often related with multiple contributing factors (inadequate nutrition, decreased physical activity, emotional/behavioural and psychological factors), but the exact pathomechanism is still unknown.

In 2006 the Rome III uniform diagnostic criteria were released, and according to them a patient must have experienced at least two of the listed symptoms during the preceding month. The list of Rome III criteria for preschool children are summarised in Table I.

\section{Aetiology and risk factors}

Habitual constipation as a result of voluntary and regular stool holding should be distinguished from other types of constipation, which are associated with organic disorders. Although this type of constipation is the most common, the diagnosis can be made only after excluding recognised organic causes $[3,4]$. The principal organic causes of constipation in children are presented in Table II.
Chronic stool holding by toddlers is usually related with a history of painful defecation (most often between 18 months and 36 months of age). At that time young children learn voluntary control through the process of toilet/potty training, and the passage of large, hard stools that painfully stretch the anus may frighten them. Other important risk factors include the following: chronic constipation during infancy, prematurity, improper psychological development, positive family history, lowered muscle tone, male sex (especially in early infancy and at pre-school age), cow's milk intolerance, inadequate nutrition (diet poor in fibre, rich in fats and sugars, sweet drinks), low level of physical activity, and, finally, psychological and behavioural factors.

The psycho-emotional background of functional constipation is usually associated with fears and phobias due to changes in surroundings and routine. Common examples are starting/changing nursery, toilet/potty training, family problems, or, in extreme situations, sexual abuse. There is also a group of children ignoring the urge to have a bowel movement because their attention is focused on other "extremely interesting activities".

\section{Vicious circle}

The most frequent cause of habitual constipation in children is definitely painful bowel movement [5]. 
As a result, a child holds on to the stool, trying to ignore the desire to empty the bowels even for a few days. This, in turn, leads to a greater reabsorption of water through the rectal mucosa and hardening of the stool making the next bowel movement more painful.

Children who are habitually constipated get into unusual positions. For example, toddlers arch their back, stand on their tiptoes, and wriggle or fidget, or they may squat, hiding in a corner.

The vicious circle mechanism evokes and escalates additional non-specific symptoms such as abdominal distension, excessive postprandial fullness, loss of appetite, encopresis, blood and mucus in the stool, nausea, vomiting, and abnormally slow weight gain. First and foremost, this mechanism generates and enhances the fear of defecation.

\section{Evaluation}

The evaluation process includes three classic stages: interview, physical examination, and medical tests. At each stage it is important to remember about differential diagnosis: functional vs. organic constipation.

The interview should include all of the possible risk factors, detailed symptoms and behavioural changes, developmental history, and psycho-social determinants such as social and sanitary conditions, and any problems in nursery/preschool or in the family.

The physical examination should include the assessment of psychological and physical development (using centile rank), and examination of the perianal area, which may show an abnormally placed external sphincter, possible anal fistules/fissures, inflammation, and signs indicating sexual abuse. The presence of $a b$ dominal distension, tenderness, and often palpable faecal masses in the abdominal examination is another important clinical feature that should not be overlooked.

The rectal examination should assess anal tone, size of rectum, and the amount and consistency of stool within the rectum. In contrast to organic causes, functional constipation is correlated with a strong fear of rectal examination.

Additionally, some patients require neurological consultation, including functional assessment of the lower part of the spine.

Medical tests are most often ordered for patients with clinical suggestions of an organic disease (based on interview and physical examination) or for children who fail to respond to the conventional therapy.

Until recently, plain abdominal X-ray was the imaging procedure for children who vigorously resist rectal examination. However, low correlation between clinical and radiological appearance made abdominal X-ray less
Table I. Paediatric Rome III criteria for constipation

Rome III criteria for functional constipation in infants up to 4 years of age

1. Fewer than 2 defecations per week

2. At least one episode of faecal incontinence per week

3. History of retentive posturing or excessive volitional stool retention

4. History of painful or hard bowel movements

5. Presence of a large faecal mass in the rectum

6. History of large diameter stools

Accompanying symptoms may include irritability, decreased appetite, and/or early satiety. The accompanying symptoms disappear immediately following passage of a large stool.

Table II. Principal organic causes of constipation in children

\begin{tabular}{ll}
\hline Anatomical causes & - Imperforate anus \\
& - Anal stenosis \\
& - Spinal cord abnormalities \\
\hline Gastrointestinal causes & - Hirschsprung's disease \\
& - Anal malformations \\
& - Intestinal neuronal \\
& dysplasia \\
\hline Endocrine and metabolic & - Hypoparathyroidism \\
causes & - Hiabetes mellitus \\
& - Hypokalaemia \\
- Intoxication with vit. D & - Gluten enteropathy \\
\hline Drugs & Opiates \\
& - Anticholinergics \\
& - Antidepressants \\
\hline - Antiepileptics
\end{tabular}

useful [6], and now the X-ray has been replaced by abdominal ultrasound.

Colonic transit studies allow physicians to distinguish between functional constipation with normal peristalsis, and constipation with delayed colonic transit and abnormal segmental contractions [7].

Ano-rectal manometry: undoubtedly the most important benefit of this examination is the ability to confirm/rule out Hirschsprung's disease by measuring the parameters of recto-anal inhibitory reflex (RAIR). The RAIR is a transient relaxant response of the internal anal sphincter to the rectal distension that normally occurs but is absent in Hirschsprung's disease. Some authors, however, indicate that RAIR cannot fully confirm normal autonomic innervation of the colon and, for this reason, biopsy is sometimes necessary $[8,9]$. 
Other medical tests include the following: colonic manometry, electromyography of the pelvic floor muscles, defecography, and dynamic nuclear magnetic resonance (NMR).

\section{Management}

The length of treatment varies, and it may take from a few months up to as long as 4 years $[10,11]$. That is why, in order to be effective, the therapy requires extreme discipline and well-coordinated activities of all "members of the team": the little patient, parents/ guardians, and physician. In many cases the intervention of a consulting psychiatrist and psychologist is necessary for success, and sometimes, when serious emotional and family problems develop, even constant psychological, psychiatric, and counselling care is recommended.

The process of management includes non-pharmacological and pharmacological interventions (Table III). The algorithm of management of constipation in children according to Rajindrajith and Devanarayana [10] is presented in Figure 1.

When functional constipation is finally recognised, a specific management program must be individually adapted for each patient to enable its implementation according to the patient's needs and preferences. Usually such a program consists of six steps: the evacuation of faeces/faecal stones accumulated in the rectum (when necessary), a change in dietary habits, toilet/ potty training, behavioural treatment, family support, and pharmacotherapy.

Evacuation of faeces/faecal stones accumulated in the rectum - not all patients require invasive interventions (administration of enemas, or mechanical evacuation). This management is recommended for children with chronic stool retention that may lead to symptoms of intestinal obstruction. According to Fishman et al., less than $5 \%$ of children necessitate intensive evacuation of faecal mass using enemas [12].

Change in dietary habits - most often this is a general part of effective therapy of functional constipation. A well-balanced diet that treats and prevents constipation should include the following:
- Fibre-rich foods. Fibre stimulates gastrointestinal peristalsis, softens stools by absorbing water, and increases colon sensitivity to mechanical stimuli. Fibre-rich foods consumed at a predictable time each day guarantee positive effects of management. The daily dose can be calculated according to the formula: age (in years) $+5=$ number of grams of fibre/day [13]. The dose of fibre must be estimated individually for each patient and gradually increased by quarter-teaspoon increments until daily recommendations for fibre are reached and/or until stools are soft. However, when the fibre administration is discontinued, all of the beneficial effects are lost.

- Control of fluid intake. Holiday and Segar recommendations are among the most useful methods for calculating total fluid intake (in $\mathrm{ml}$ ) for children. For children with body weight $1-10 \mathrm{~kg}=100 \mathrm{ml} / \mathrm{kg}$, for children with body weight $11-20 \mathrm{~kg}=1000 \mathrm{ml}+50 \mathrm{ml} / \mathrm{kg}$ for every $\mathrm{kg}$ over $10 \mathrm{~kg}$ of body weight, for children with body weight above $20 \mathrm{~kg}=1500 \mathrm{ml}+20 \mathrm{ml}$ for every kilogram above $20 \mathrm{~kg}$ of body weight. The daily ingestion of milk should be limited (depending on the child's age) to 230-350 ml. Fruit juices should be limited to no more than $120 \mathrm{ml} /$ day and replaced with fresh and dried fruit (apple in pieces and plums) and vegetables. The rest of the fluid requirement should be replaced with pure water [14].

- Avoidance of foods high in fat and sugar, fast foods, fried foods, and sweet drinks (especially carbonated beverages). Decrease in ingestion of white bread, potatoes, rice, and fruits that may cause constipation (bananas, boiled and grated apple). Increased intake of whole grains bread, groats, fruits, and vegetables known to treat constipation (pears, broccoli, apples in pieces, carrots, beans, pineapple).

- Use of herbs and spices - dill tea, anise, chamomile tea, olive oil (added to soup), water with honey in the morning (honey for children above the age of 2 years).

- Any nutritional change should be accompanied with increased healthy and regular physical activity including daily outdoor exercise, and reduced screen time (TV, computer) to a maximum of $2 \mathrm{~h} /$ day [15].

Table III. Non-pharmacological and pharmacological management of functional constipation in children

\begin{tabular}{|c|c|}
\hline Non-pharmacological management & Pharmacological management \\
\hline $\begin{array}{l}\text { - Education } \\
\text { - Behavioural treatment } \\
\text { - Biofeedback (less effective in habitual constipation) } \\
\text { - Diet rich in fibre } \\
\text { - Increased fluid intake } \\
\text { - Exercise } \\
\text { - Psychotherapy }\end{array}$ & $\begin{array}{l}\text { - Osmotic laxatives (magnesium hydroxide, magnesium sulphate, } \\
\text { lacticol, lactulose, glycerine suppositories) } \\
\text { - Stimulants (bisacodyl, castor oil) } \\
\text { - Softeners (docusate sodium, liquid paraffin) } \\
\text { - Bulk-producing agents (methylcellulose, dietary fibre, psyllium) } \\
\text { - Serotonin receptor agonists (Tegaserod) } \\
\text { - Spasmolytics (Trimebutine) } \\
\text { - Probiotics (data still conflicting) }\end{array}$ \\
\hline
\end{tabular}




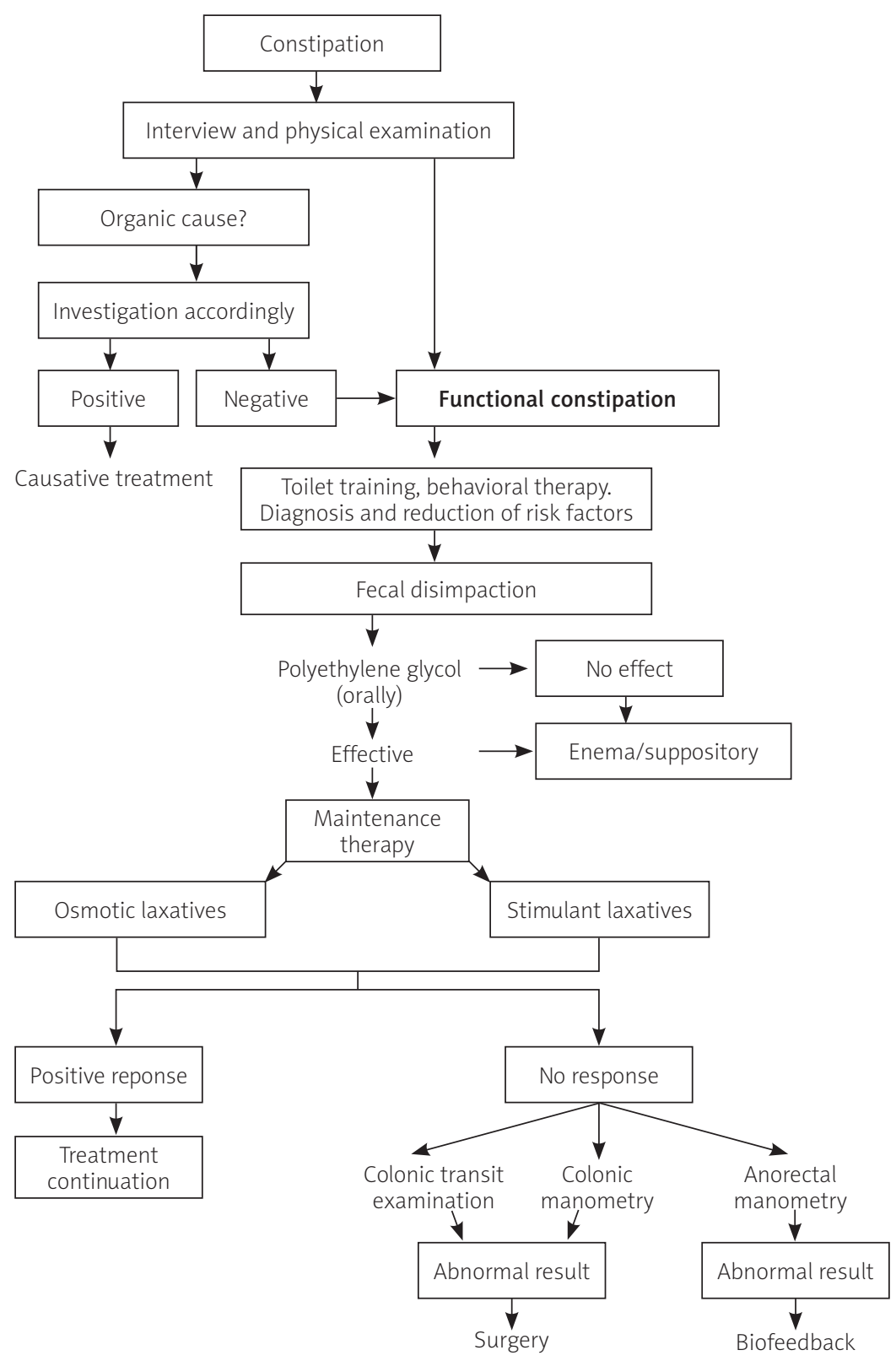

Figure 1. Algorithm of management of constipation in children according to Rajindrajith and Devanarayana

- Probiotics as dietary supplements. Data about the beneficial effects of probiotics in constipated children are still conflicting. Some authors confirm their positive influence on motor activity in the gastrointestinal system [16], while others report that probiotics are not effective as adjuncts in treating functional constipation [17]. To develop a uniform consensus, more randomised, double-blind, placebo-controlled studies on large study groups are needed.

Toilet/potty training - this means a regular process of training a young child to use the toilet/potty. There is a natural reflex mechanism (gastrocecal, gastrocolic, and duodenocolic reflexes), which stimulates the urge to defecate and which works best 20-30 min after a main meal.

To strengthen pelvic floor muscles, the child should also learn how to urinate with an interrupted urine stream. Any "toilet/potty success" should be rewarded for positive reinforcement.

The toilet/potty training is useless until a child has control over the bowel and bladder muscles and is familiar with the toilet/potty seat.

Behavioural treatment - its role is to help a child to understand and realise that defecation is a routine activity, and to encourage a child to stop stool holding. 
This method is based on increased awareness and lowered fear of defecation.

Behavioural treatment uses developmentally appropriate strategies, such as pictures, stories, and demonstration games (teaching proper position, holding breath, using relaxation techniques). The little reward system, as a positive stimulation, works well for maintenance of a stool diary.

Family support. Family members need to be educated for additional advantages of treatment and to avoid mistakes in the whole therapy process (e.g. if family members do not follow dietary instructions, or, something that is totally unacceptable, if any rectal manipulations, such as a thermometer or other object insertion, are practiced).

Pharmacological treatment - should be considered as a maintenance therapy. The most popular medications are oral laxatives (lactulose, lacticol, and polyethylene glycol). Other substances are summarised in Table III.

Undoubtedly, the most effective and recommended method of treatment is nonpharmacological intervention, which is safe, has no side effects, and brings long-term benefits. Unfortunately, some children fail to respond to this management and they require pharmacological treatment.

\section{Case study}

A 22-month-old boy was brought to the family doctor's office by his mother, who was concerned that her child had been having painful bowel movements every 4 days for about 1 month. The child was born at term by spontaneous vaginal delivery without any complications and had ten Apgar points after birth. On physical examination he appeared normal, developing well on his growth curves. His medical history showed cow's milk intolerance and temporary constipation in infancy (within the $7^{\text {th }}$ month of his life, after breastfeeding cessation). The child attended nursery school from the age of 12 months and started potty training when he was 18 months old. Based on his history, his diet contained an insufficient amount of fresh vegetables and fruit because he refused to eat homemade baby food rich in natural nutrients (almost all fruit and vegetables were from jar food). His mother reported problems with potty training because the child was not showing any interest in the potty and was "too busy" to be trained.

Physical examination revealed a well-nourished and cheerful child, with normal vital signs and physical and psychomotor development, except for slight abdominal distension, without any pain or tenderness. The perianal region remained unchanged.

After careful evaluation and exclusion of organic diseases, the child was put on an individual treat- ment plan. The therapy included nonpharmacological intervention that was aimed towards dietary and behavioural modifications, with administration of lactulose $(3 \times 5 \mathrm{ml} / \mathrm{day})$ and occasionally trimebutine $(2 \times 5 \mathrm{ml} /$ day $)$. Mechanical removal, or removal stimulated by enema evacuation of faecal loading was not necessary. Nutritional changes included adding yogurt with fruit and ground porridge oats once a day (homemade meal). The dose of oats was estimated individually and was increased by half a teaspoon until the expected effect occurred. Additional modification included a portion of raw salad added to the main meal and increased fluid intake (water with honey and lemon, compote from dried apricots and plums, without sugar). The mother was instructed on how to make potty training fun by using little rewards system, and she was asked to increase outdoor activities by at least $1 \mathrm{~h}$ per day.

Within 3 weeks, the patient's mother reported that her son was having regular bowel movements with a frequency of once every 2 days, but still accompanied with severe straining. The maintenance therapy was continued for the following few weeks until a scheduled control visit.

\section{References}

1. Van den Berg MM, Benninga MA, Di Lorenzo C. Epidemiology of childhood constipation: a systematic review. Am J Gastroenterol 2006; 101: 2401-9.

2. Sonnenberg A, Koch T. Physician visits in the United States for constipation: 1958 to 1986. Dig Dis Sci 1989; 34: 606-11.

3. Levine M. Children with encopresis: a descriptive analysis. Pediatrics 1975; 56: 412-6.

4. Youssef $N$, DiLorenzo $C$. The role of mobility in functional abdominal disorders in children. Pediatr Ann 2001; 30: 24-30.

5. Borowitz SM, Cox DJ, Tam A, et al. Precipitant of constipation during early childhood. J Am Board Fam Pract 2003; 16: 213-8.

6. Reuchlin-Vroklage LM, Bierma-Zeinstra S, Benninga MA, et al. Diagnostic value of abdominal radiography in constipated children: a systematic review. Arch Pediatr Adolesc Med 2005; 159: 671-8.

7. Cook BJ, Lim E, Cook D, et al. Radionuclear transit to assess sites of delay in large bowel transit in children with chronic idiopathic constipation. J Pediatr Surg 2005; 40: 478-83.

8. Kaur G, Gardiner A, Duthie GS. Rectoanal reflex parameters in incontinence and constipation. Dis Colon Rectum 2002; 45 : 928-33.

9. Meunier P, Marechal JM, Mollard P. Accuracy of the manometric diagnosis of Hirschsprung's disease. J Pediatr Surg 1978; 13: 411-5.

10. Rajindrajith S, Devanarayana NM. Constipation in children: novel insight into epidemiology, pathophysiology and management. J Neurogastroenterol Motil 2011; 17: 35-47. 
11. Czerwionka-Szaflarska M, Romańczuk B. Management of functional constipation stools in children and adolescents [Polish]. Forum Med Rodz 2008; 2: 349-57.

12. Fishman L, Rappaport L, Schonwald A, et al. Trends in referral to a single encopresis clinic over 20 years. Pediatrics 2003; 111: 604-7.

13. Mason D, Tobias N, Lutkenhoff M, et al. The APN's guide to pediatric constipation management. The Nurse Practitioner 2004; 29: 13-21.

14. Holiday MA, Segar WE. The maintenance need for water in parenteral fluid therapy. Pediatrics 1957; 19: 23-32.

15. Coehlo DP. Encopresis: a medical and family approach. Pediatric Nursing 2011; 37: 107-12.

16. Bekkali NL, Bongers ME, Van den Berg MM, et al. The role of probiotics mixture in the treatment of childhood constipation: a pilot study. Nutr J 2007; 6: 17.

17. Banaszkiewicz A, Szajewska H. Ineffectiveness of Lactobacillus GG as an adjunct to lactulose for the treatment of constipation in children: a double blind placebo controlled randomized trial. J Pediatr 2005; 146: 363-8.

Received: 15.03 .2012

Accepted: 12.07 .2012 